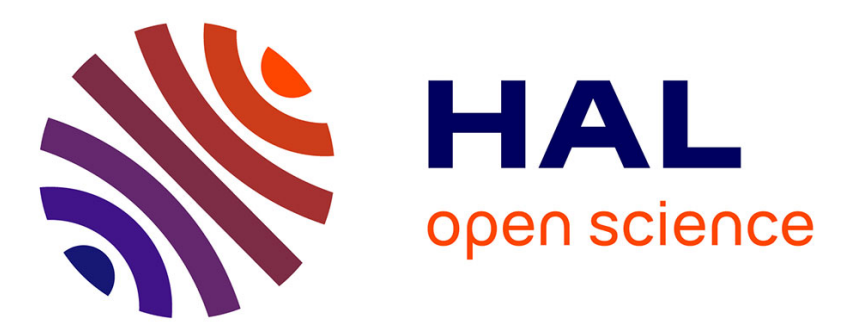

\title{
Influence de l'introduction de défauts colonnaires amorphes sur les propriétés de transport d'un monocristal supraconducteur à haute Tc
}

Franck Warmont, Sylvie Hébert, Vincent Hardy, Christine Martin, Charles Simon, Jackie Provost

\section{To cite this version:}

Franck Warmont, Sylvie Hébert, Vincent Hardy, Christine Martin, Charles Simon, et al.. Influence de l'introduction de défauts colonnaires amorphes sur les propriétés de transport d'un monocristal supraconducteur à haute Tc. Journal de Physique III, 1997, 7 (12), pp.2385-2392. 10.1051/jp3:1997266 . jpa-00249727

\section{HAL Id: jpa-00249727 https://hal.science/jpa-00249727}

Submitted on 1 Jan 1997

HAL is a multi-disciplinary open access archive for the deposit and dissemination of scientific research documents, whether they are published or not. The documents may come from teaching and research institutions in France or abroad, or from public or private research centers.
L'archive ouverte pluridisciplinaire HAL, est destinée au dépôt et à la diffusion de documents scientifiques de niveau recherche, publiés ou non, émanant des établissements d'enseignement et de recherche français ou étrangers, des laboratoires publics ou privés. 


\title{
Influence de l'introduction de défauts colonnaires amorphes sur les propriétés de transport d'un monocristal supraconducteur à haute $T_{\mathbf{c}}\left(^{*}\right)$
}

\author{
Franck Warmont, Sylvie Hébert, Vincent Hardy, Christine Martin, Charles Simon
} and Jackie Provost $\left({ }^{* *}\right)$

Laboratorre CRISMAT $\left({ }^{* * *}\right)$, ISMRA, Université de Caen, 14050 Caen, France

(Reçu le 8 juillet 1997, révisé le 20 octobre 1997, accepté le 21 octobre 1997)

\begin{abstract}
PACS.74 25.Fy - Transport properties (electric and thermal conductivity, thermoelectric effects, etc)

PACS.74.60 Ge - Flux pinning, flux creep and flux-lme lattice dynamics

PACS.74 $72 \mathrm{Fq}$ - Tl-based cuprates
\end{abstract}

Résumé. - L'irradiation aux ions lourds de haute énergie permet d'introdurre des défauts colonnaires amorphes dans les supraconducteurs à haute $T_{c}$. Ces centres de pinnıng artificiel, de morphologie connue, sont introduits en concentration facıle à maîtrıser. L'efficacité de ces défauts a été très souvent démontrée à partir de mesures d'aimantation. L'étude présentée ici : mesure de la résistance selon l'axe $\mathbf{c}, R_{c}(T)$, en présence de défauts colonnarres parallèles à l'axe c, montre que ces défauts sont capables de s'opposer efficacement à l'effet des fluctuations thermiques. Les mesures ont été faltes sur le même monocristal avant et après l'rradıation.

\begin{abstract}
Columnar defects can be introduced in high $T_{\mathrm{c}}$ superconductors by irradiation with high energy heavy ions. The concentration of these artificial pinning centers with a well characterized morphology is easily controlled. The pinning efficiency of these defects has been often demonstrated. mannly from magnetization measurements. In the present work, measurements of the electrical resistance along the $\mathrm{c}$ axis, $R_{\mathrm{c}}(T)$, in the presence of columnar defects are presented. They show the ability of these defects to prevent the thermal fluctuations effects The measurements have been performed on the same crystal before and after the heavy ion irradiation.
\end{abstract}

Les Supraconducteurs à Haute Température Critique (SHTC) présentent un intérêt technologique considérable dans les domaines de l'électrotechnique et de l'électronique. En nous limitant au champ d'application de l'électrotechnique et des courants forts, deux paramètres justifient l'intérêt énorme porté à ces matériaux : leur température critique $T_{c}$ élevée, jusqu'à $133 \mathrm{~K}$ pour les cuprates de mercure et leur champ critique supérieur $B_{\mathrm{c} 2}$ très élevé. Ces caractéristiques les rendent, a prıor, utilisables dans l'azote lıquide. Cependant, jusqu'à maintenant,

$\left(^{*}\right)$ Le contenu de cet article a été présenté aux $V^{\text {èmes }}$ journées de Cryogénıe et de Supraconductıvité à Aussors en avril 1997

(**) Auteur auquel doit être adressé la correspondance (e-mail: provost@crismat.ismra fr).

$\left({ }^{* * *}\right)$ CNRS UMR 6508

(C) Les Éditions de Physique 1997 


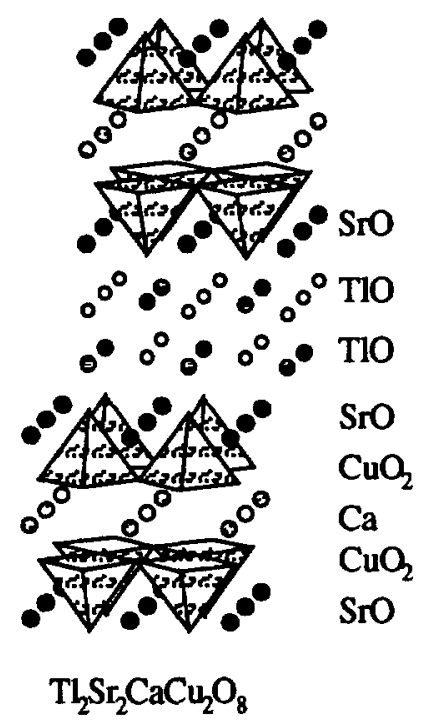

Fig. 1. - Structure de Tl-2212

[Structure of Tl-2212.]

ces matériaux n'ont pas encore d'application réellement industrielle [1] en raison d'une trop faible densité de courant critique, notamment sous champ magnétique, et de difficultés de mise en forme.

\section{Densité de courant critique}

Leur faible densité de courant critique a plusieurs origines Des origines intrinsèques comme la très faible valeur de leur longueur de cohérence $\xi_{\mathrm{c}}$ selon l'axe $\mathbf{c}$ de la structure cristalline et l'anisotropie électronique de ces composés peuvent être invoquées. Ces deux grandeurs sont reliées et résultent du caractère bidimensionnel de la structure.

Dans les composés les plus anisotropes : $\mathrm{Bi}_{2} \mathrm{Sr}_{2} \mathrm{CaCu}_{2} \mathrm{O}_{8}$ (Bi-2212) et $\mathrm{Tl}_{2} \mathrm{Ba}_{2} \mathrm{CaCu}_{2} \mathrm{O}_{8}$ (Tl-2212) (Fig 1), la très petite valeur de $\xi_{\mathrm{c}}$ entraîne un comportement bidimensionnel du réseau de vortex. Ce comportement $2 \mathrm{D}$ a été attribué [2] à un découplage des plans $\mathrm{CuO}_{2}$ supraconducteurs (plans a-b de la structure) dû à la suppression du couplage entre ces plans par rupture de l'effet Josephson sous l'action d'un champ magnétique relativement faible.

Le réseau de vortex adopterait alors un comportement $2 \mathrm{D}$, les plans adjacents formés de vortex "galettes" glissant alors facilement les uns sur les autres, donnant heu à une dissipation d'énergie, d'où une densité de courant critique $\mathbf{j}_{\mathrm{c}}$ très limitée. D'autres transitions de phase du réseau de vortex que nous ne détaillerons pas ici [3] ont été évoquées pour rendre compte des résultats expérımentaux Ces études font l'objet de recherches très actives dans le but de comprendre la dynamique des vortex dans ces matériaux et d'essayer de trouver des moyens pour accroître la densité de courant $\mathbf{j}_{\mathrm{c}}$, comparée à celle des céramiques conventionnelles. 


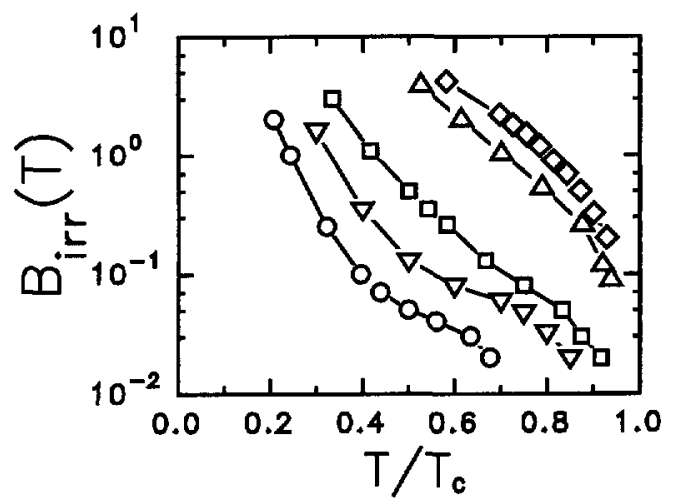

Fig 2 - Lignes d'ırréversibilité des cuprates supraconducteurs (de la plus basse vers la plus haute · Bi-2212, Tl-2212, Tl-2223, Tl-1223 et (Tl/Bi)-1212) extraites de mesures d'amantation.

[Irreversibility lines of superconducting cuprates extracted from magnetization measurements (from the lowest to the highest: Bi-2212, Tl-2212, Tl-2223, Tl-1223 et ( $\mathrm{Tl} / \mathrm{Bl}$ )-1212).]

\section{Ligne d'irréversibilité}

La fraction utile du diagramme "champ magnétique/température" adaptée pour les applications utilisant le transport de courant sans dissipation est limitée à la zone située au dessous de la Ligne d'Irréversibilité [4] (LI) (Fig. 2). Cette ligne sépare le plan $B / T$ en deux régions : au-dessous de (LI) il existe un courant critique alors qu'au-dessus de (LI) $\mathbf{j}_{\mathbf{c}}=0$, excluant toute application potentielle.

La ligne d'irréversibilité est d'autant plus basse dans le plan $B / T$ que le matériau est plus anisotrope. Cette "hiérarchie" des lignes d'irréversibilité a été mise en évidence expérimentalement [5] dans les cuprates de thallium (Fig. 2) qui présentent un grand nombre de phases cristallines avec des anisotropies variées

Le composé Tl-2212 étudié dans cet artıcle a une anisotropie élevée, voisine de celle du composé $\mathrm{Bl}-2212$, alors que Tl-1223 et ( $\mathrm{Tl} / \mathrm{Bi}$ )-1212, les moins anisotropes de la série, se rapprochent, de ce point de vue, de $\mathrm{Y}-123\left(\mathrm{YBa}_{2} \mathrm{Cu}_{3} \mathrm{O}_{7}\right)$.

La ligne d'irréversibilité peut être déterminée à partir des courbes d'almantation $M(H)$ enregistrées à différentes températures ou à partir de mesures de transport : résistance mesurée en fonction de la température $R(T)$, sous différents champs magnétiques

Le moyen le plus efficace trouvé jusqu'à maintenant pour déplacer la ligne d'irréversibilité vers les champs plus élevés et les plus hautes températures afin d'agrandir le domaine d'irréversibilité dans le diagramme $B / T$ est l'introduction de défauts colonnaires amorphes [6,7] par irradiation aux ions lourds de haute énergie.

\section{Les défauts colonnaires}

L'irradiation de matériaux aux ions lourds de haute énergie délivrés par GANIL, à Caen, permet de créer dans certains matériaux des traces cylindriques amorphes [7].

Dans les SHTC, on obtient des traces amorphisées ou "défauts colonnaires" si l'énergie déposée par excitations électroniques, $(-\mathrm{d} E / \mathrm{d} x)_{\mathrm{el}}$, dans le matériau cible dépasse une valeur seuil 


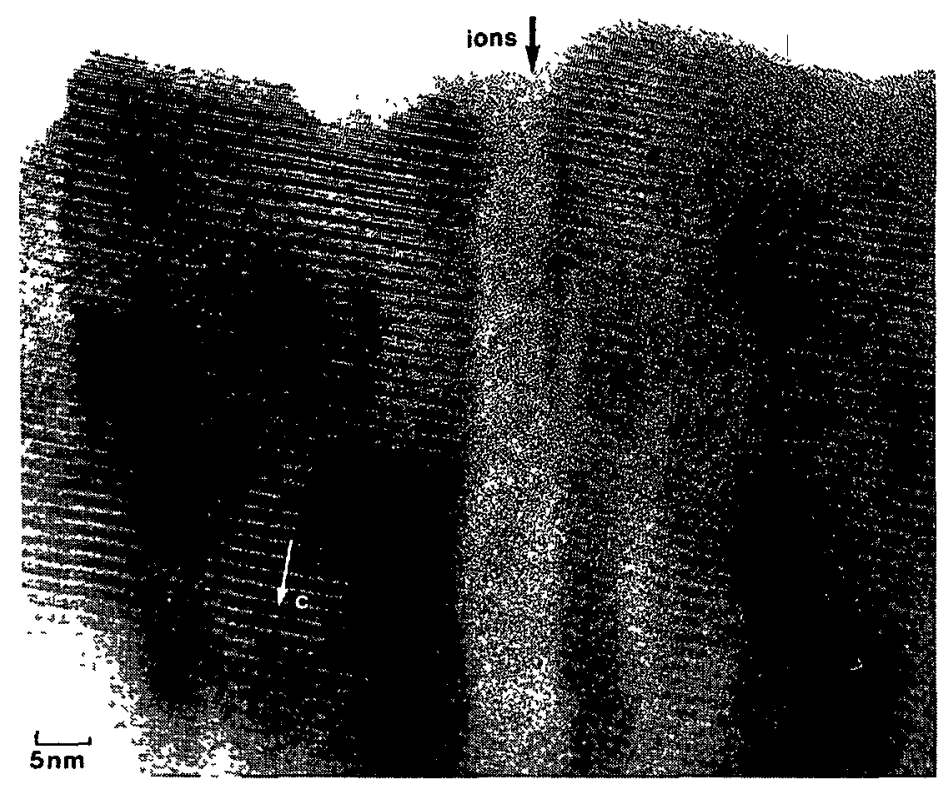

Fig. 3. - Défaut colonnaire amorphe continu dans une céramıque Tl-2212 (ion $\mathrm{Pb}$ de $6 \mathrm{GeV}$ ).

[Continuous amorphous columnar defect in a $\mathrm{Tl}-2212$ ceramic (6 GeV Pb ion).]

Avec les ions $\mathrm{Pb}$ de $6 \mathrm{GeV}$ utilisés lors de l'expérience décrite ici, on obtient, dans tous les oxydes de cuivre supraconducteurs, des défauts colonnaires amorphes de diamètre compris entre 7 et $10 \mathrm{~nm}$, selon le matériau particulier. Un exemple de défaut colonnaire, observé dans un grain d'une céramique (Fig. 3) illustre leurs dimensions nanométriques.

Leur diamètre est comparable au diamètre du cœur normal d'un vortex et leur symétrie est la même. Ces deux caractéristiques en font des centres d'ancrage des vortex dont l'efficacité a été largement prouvée [7].

\section{But de l'expérience}

Comme nous l'avons indiqué ci-dessus, dans les matériaux les plus bidimensionnels, du fait de la modulation du paramètre d'ordre selon c, les vortex adoptent un comportement $2 \mathrm{D}$ dès que le champ appliqué augmente. Dans ces conditions les fluctuations thermiques sont susceptibles d'accélérer la disparition du comportement "ligne" des vortex et de les transformer en vortex "ondulés" se déplaçant quasi librement dans le cristal, entraînant une dissipation.

Nous avons voulu vérifier si la présence de défauts colonnaires pouvait, par une sorte d'effet d'alignement, retarder l'effet des fluctuations thermiques. Le meilleur matériau candidat pour une telle étude était, a prior, le Bi-2212 puisque le plus anisotrope (voir la hiérarchie des (LI) de la Fig. 2) ; le second étant le Tl-2212. Le composé B1-2212 présentait en outre l'avantage d'être synthétisé sous forme de monocristaux ayant des dimensions transversales (plans a-b) plus grandes que celles des cristaux de Tl-2212. Les deux types de monocristaux sont synthétisés au sein du laboratoire CRISMAT et présentaient à cet égard les mêmes avantages Bien qu'intrinsèquement moins favorable, nous avons cependant choisi $\mathrm{Tl}_{-}-2212$ car les monocristaux sont légèrement plus épais que ceux de $\mathrm{B} 1-2212$ et ils ont une tenue mécanique bien supérieure.

Il est en effet connu que les cristaux de $\mathrm{Bi}-2212$ clivent facilement et que, dans ces conditions, 


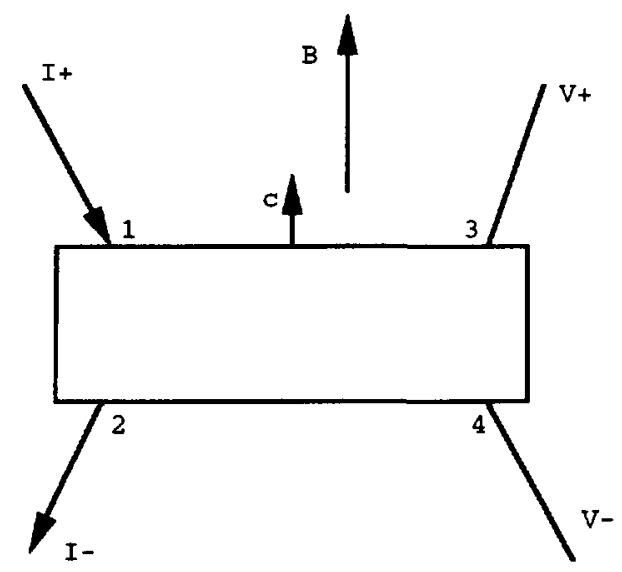

Fig. 4. - Mesure $R_{c}(T)$ en configuration "cross".

$\left[R_{\mathrm{c}}(T)\right.$ measurement in the "cross" configuration.]

un léger décollement des plans $\mathrm{CuO}_{2}$ peut se produire lors de la manipulation des échantillons. Cette modification peut affecter la résistance électrique selon l'axe c $R_{\mathrm{c}}(T)$ mesurée lors de l'étude rapportée ici.

\section{La procédure d'irradiation}

Les monocristaux de $\mathrm{Tl}-2212$ ont été irradiés à la température ambiante avec des ions $\mathrm{Pb}$ de $6 \mathrm{GeV}$ produisant des défauts colonnaires amorphes traversant l'échantillon de part en part. L'épaisseur des cristaux était, en effet, nettement inférieure au parcours des ions dans ce matériau. Le faisceau d'ions incidents était parallèle à l'axe $\mathbf{c}$ du cristal. Le flux $\phi$ d'ions a été limité à $5 \times 10^{7}$ ions $/ \mathrm{cm}^{2} \mathrm{~s}$, des expériences effectuées antérieurement ayant montré qu'un tel flux n'entraîne pas d'échauffement notable des cristaux. L'irradiation a été arrêtée lorsque la densité surfacique de défauts colonnaires, ou fluence $\phi t$ a atteint $10^{11}$ ions $/ \mathrm{cm}^{2}$. Pour cette densité de défauts colonnaires, on peut admettre que le recouvrement des traces est négligeable et donc, que chaque ion traversant le matériau crée un défaut colonnaire amorphe.

Le faisceau d'ions de GANIL conduit à une distribution aléatoire d'impacts sur la face d'entrée des cristaux (distribution de Poisson). Nous introduirons, comme d'habitude dans ce type d'expérience sur les SHTC, un champ équivalent de fluence, $B_{\phi}$, champ moyen qui existerait dans le cristal si chaque défaut colonnaire était occupé par un vortex :

$$
B_{\phi}=\phi t \phi_{0}
$$

où $\phi_{0}$ est le quantum de flux.

Signalons enfin que les cristaux ont été irradiés avec les fils d'amenée de courant et de mesure de potentiel soudés et mis en place de sorte que, entre les mesures effectuées avant l'irradiation et après l'irradiation les cristaux n'ont subi aucun autre traitement susceptible d'altérer leurs propriétés. 


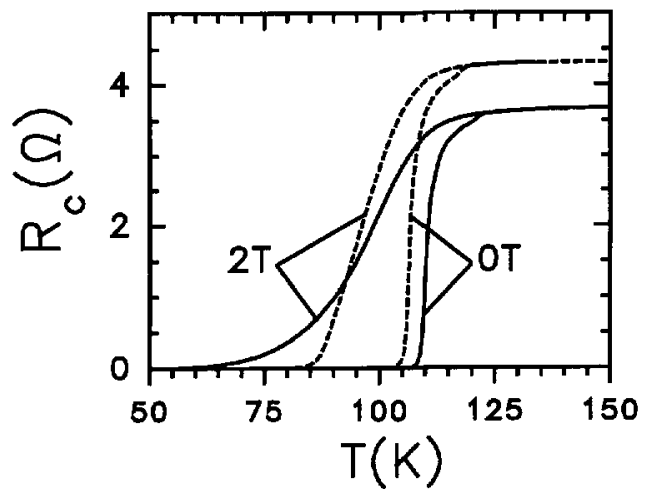

Fig 5 - Résistance en fonction de la température sous champ nul et sous $2 \mathrm{~T}$ avant et après irradiation. (traits continus avant irradiation).

[Electrical resistance versus temperature under zero field and under $2 \mathrm{~T}$ before and after irradiation. (continuous line: before irradiation).]

\section{Mesure de la résistance $R_{c}(T)$}

La mesure de la résistance électrique a été effectuée en adoptant la configuration de contacts dite "cross" représentée sur la figure 4. Les contacts 1 et 2 situés sur les faces opposées du cristal servent à l'alimentation en courant. La tension est mesurée entre les contacts 3 et 4 qui sont également situés de part et d'autre du cristal.

Comme il s'agit icı de comparer la résistance selon l'axe c avant et après l'irradiatıon, sur le même cristal. les résultats expérimentaux n'ont pas été analysés en terme de résistivité $\rho_{\mathrm{c}}$. Les valeurs de $R_{\mathrm{c}}(T)$ rapportés ici sont donc les valeurs du rapport $R=V / I$ en ohms.

Les mesures ont été effectuées dans un cryostat "Oxford Instruments" équipé d'une bobine supraconductrice dans la gamme de champ allant de 0 à 7 teslas.

Pour étudier l'effet des fluctuations, nous avons adopté une configuration "sans force de Lorentz" puisque lors des mesures, le courant $I$ et le champ magnétique $B$ étaient parallèles (et parallèles à l'axe c du cristal). Dans ces conditions, les modifications du régime de dissipation peuvent être reliées au changement de régime des fluctuations.

\section{Résultats et discussion}

Sur la figure 5 nous avons reporté $R_{\mathrm{c}}$ en fonction de la température pour le même cristal, avant et après irradiation à $\phi t=10^{11}$ ions $/ \mathrm{cm}^{2}$, sous champ nul et sous $B=2$ teslas.

On remarque que la résistance du cristal dans l'état normal augmente après l'irradiation. Cette augmentation est liée à l'introduction de défauts supplémentaires dans le réseau cristallin Sous champ nul la température à laquelle la résistance s'annule, $\left(T_{\mathrm{C}_{\mathrm{offset}}}\right)$ diminue après l'irradiation, de $3 \mathrm{~K}$ environ.

Ce type de diminution est très généralement observé pour des HSTC irradiés aux ions lourds, à cette fluence.

Sous champ magnétique de 2 teslas, les courbes $R_{\mathrm{C}}(T)$ obtenues avant et après irradiation se croisent entre 90 et $95 \mathrm{~K}$ et la température $T_{\mathrm{c}_{\text {offset }}}$ est, cette fors, plus basse pour l'échantillon non irradié. 


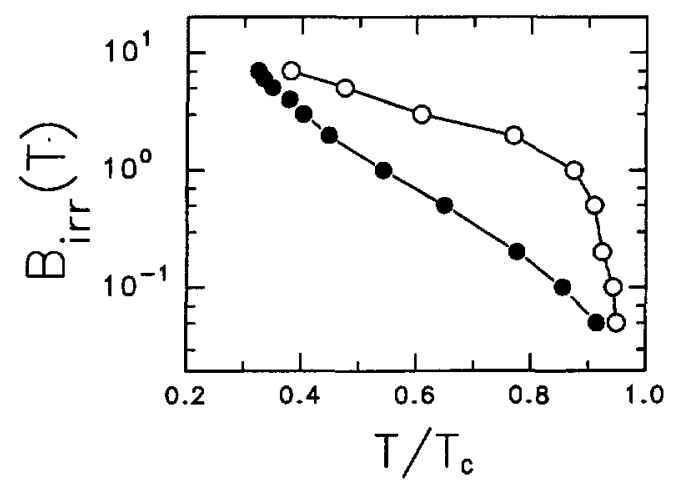

Fig 6 - Ligne d'ırréversibılıté déterminée par mesures de transport avant irradiation (ronds plems) et après irradiation (ronds vides).

[Irreversibility line extracted from transport measurements before irradiation (full circles) and after irradiation (empty circles).]

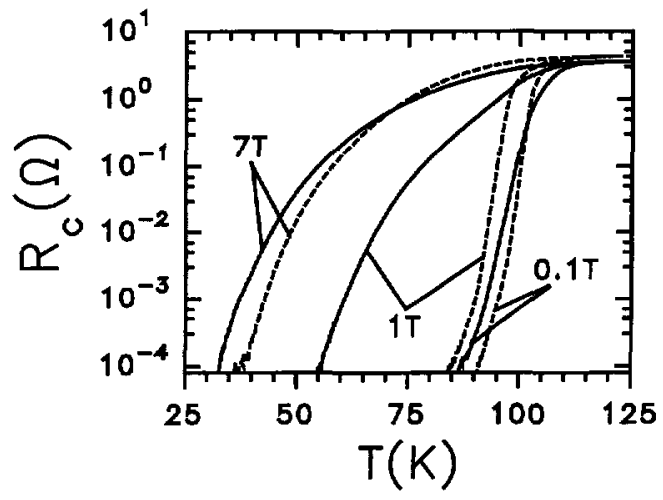

Fig. $7-R_{c}(T)$ en échelle logarithmique sous 0.1. 1 et $7 \mathrm{~T}$ Courbes en traits plems avant irradiatıon. $\left[R_{\mathrm{c}}(T)\right.$ in a logarithmic scale under $0.1,1$ et $7 \mathrm{~T}$. Curves in full lines before irradiation.]

Cette température correspond, en fait, à un niveau de résistance faible $\left(R_{\mathrm{c}}<10^{-4} \mathrm{ohm}\right)$. Cette température, sous un champ donné, sera prise comme température d'irréversibilité. Elle a été utilisée pour tracer la ligne d'irréversibilité de la figure 6, avant et après irradiation. On remarquera que la ligne d'irréversibilité du cristal non irradié est différente de la (LI) du Tl2212 reportée sur la figure 2 en raison de la différence des temps caractéristiques des méthodes de mesure (mesure d'aimantation dans le cas de la Fig. 2).

On remarque que cette (LI) présente un plateau dans la gamme des températures intermédiaires. Ce plateau est situé à $B$ de l'ordre de $B_{\phi}$ comme dans le cas de Bı-2212 [7]. La (LI) présente un coude marqué à une température réduite $t=T / T_{c}$ voisine de $t=0,85$. Cette température peut être interprétée comme la température de dépiégeage des vortex, $T_{1}$, à partir de laquelle, sous l'effet des fluctuations, les vortex peuvent sortir d'un défaut colonnaire.

Sur la figure 7 , la résistance $R_{\mathrm{c}}(T)$, portée en échelle logarithmique, a été tracée pour 3 valeurs du champ magnétique $: B=0,1 \mathrm{~T} ; 1 \mathrm{~T}$ et $7 \mathrm{~T}$.

La valeur inférieure : $0,1 \mathrm{~T}$, se situe au-dessous de $B_{\phi}$ et l'on retrouve, avant et après 
irradiation, des comportements similaires à ceux de la figure 5 à 2 teslas : augmentation de la résistance dans l'état normal et augmentation de $\mathcal{T}_{\mathrm{c}_{\text {offset }}}$ après irradiation.

Sous $B=1 \mathrm{~T}$, du même ordre de grandeur que $B_{\phi}$ et sensiblement égal au champ correspondant au coude observé sur (LI) à $t=0,85$, les courbes avant et après irradiation se croisent. On observe, comme à $B=2 \mathrm{~T}$, une augmentation très nette de $T_{\mathrm{c}_{\text {offset }}}$ après irradiation.

Enfin, à $B=7 \mathrm{~T}$, on observe encore ce comportement bien que moins prononcé qu'à $B=1 \mathrm{~T}$. Les courbes à 7 teslas montrent que la présence des défauts colonnaires s'oppose encore à l'effet des fluctuations thermiques, bien que ce champ soit nettement supérieur à $B_{\phi}$. Cette persistance des effets des défauts colonnaires au dessus de $B_{\phi}$ à déjà été signalée [8], elle montre que le comportement collectif des vortex doit être pris en compte pour interpréter plus finement les résultats expérimentaux.

\section{Conclusion}

Ces mesures de $R_{\mathrm{c}}(T)$, dans une configuration "sans force de Lorentz" montrent très clairement que la présence de défauts colonnaires peut s'opposer très efficacement à l'effet des fluctuations thermiques, même dans un milieu aussi anisotrope que Tl-2212. La persistance de ces effets au delà du champ de fluence $B_{\Phi}$ confirme bien l'efficacité de ces défauts et leur intérêt comme système "modèle" pour les études portant sur la dynamique des vortex.

\section{Bibliographie}

[1] Laumond Y., La supraconductivité et l'électronique, Rev. Gen. Froid, Novembre 1995, pp. 24-32.

[2] de Rango P., Giordanengo B., Tournier R., Sulpice A., Chaussy J., Deutscher G., Genicon J L., Lejay P., Retoux R. and Raveau B., The "irreversibility line" of $\mathrm{Bi}_{2-x} \mathrm{~Pb}_{x} \mathrm{Sr}_{2} \mathrm{Ca}_{2} \mathrm{Cu}_{3} \mathrm{O}_{10}$ : a possible breakdown of an intrinsic proximity effect., J. Phys. France 50 (1989) 2857.

[3] Zech D., Lee S.L., Keller H., Blatter G, Kes P.H and Li. T.W., Phase diagram of $\mathrm{Bi}_{2}{ }_{15} \mathrm{Sr}_{185} \mathrm{CaCu}_{2} \mathrm{O}_{8+\delta}$ in the presence of columnar defects, Phys. Rev. B 54 (1996) 6129.

[4] Müller K.A., Takashige M. and Bednorz J.G., Flux trapping and superconductive glass state in $\mathrm{La}_{2} \mathrm{CuO}_{4-y}$ : Ba., P.R.L. 58 (1987) 1143.

[5] Maignan A, Hardy V., Martin C., Pelloquin D., Villard G. and Wahl A., On the role of anisotropy in superconducting cuprates: A complete study of $\mathrm{Bi}, \mathrm{Hg}$, Tl-single crystals, MOS96, J. Low Temp. Physıcs 105 (1996) 1005.

[6] Hardy V., Provost J, Groult D., Hervieu M., Raveau B., Durčok S., Pollert E., Frison J.C., Chaminade J.P. and Pouchard M., Strong shift of the irreversibility line in bismuth and thallium based 2212 HTSC single crystals irradiated by $6.0 \mathrm{GeV} \mathrm{Pb}$ ions., Physıca $\mathbf{C}$ 191 (1992) 85.

[7] Hardy V., Provost J., Groult D., Simon Ch., Hervieu M. and Raveau B., Columnar defects induced by high energy heavy ions in HTSC. Their effect on irreversibility line and pinning properties., J Alloys and Compounds 195 (1993) 395.

[8] Steel D.G., Hettinger J.D., Parkman J.T., Yoder M.E., Gray K.E, Glagola B.G., Eddy M.M., Importance of vortex interactions in $\mathrm{Tl}_{2} \mathrm{Ba}_{2} \mathrm{CaCu}_{2} \mathrm{O}_{8}$ epitaxial films with columnar defects, Physica C265 (1996) 159. 